\title{
Nontypeable Haemophilus influenzae and Streptococcus pneumoniae bind respiratory syncytial virus glycoprotein
}

\section{Correspondence \\ Elisabeth Adderson \\ Elisabeth.Adderson@stjude.org}

Received 17 November 2006

Accepted 18 April 2007

\author{
Vasanthi Avadhanula, ${ }^{1,2}$ Yan Wang, ${ }^{1}$ Allen Portner ${ }^{1,2}$ \\ and Elisabeth Adderson ${ }^{1,2,3}$
}

\author{
${ }^{1}$ Department of Infectious Diseases, St. Jude Children's Research Hospital, Memphis, TN 38105, \\ USA \\ ${ }^{2}$ Department of Molecular Sciences, University of Tennessee Health Sciences Center, Memphis, \\ TN 38163, USA \\ ${ }^{3}$ Department of Pediatrics, University of Tennessee Health Sciences Center, Memphis, TN 38163, \\ USA
}

\begin{abstract}
Respiratory syncytial virus (RSV) infection is associated with secondary bacterial infections caused by nontypeable Haemophilus influenzae (NTHi) and Streptococcus pneumoniae. The pathogenesis of these complications is not completely understood; however, viral infection of respiratory epithelial cells promotes colonization by these bacteria. In the present study, RSV virions associated with NTHi and pneumococci in an inoculum-dependent manner in a fluid-phase binding assay. Adherence of NTHi and $S$. pneumoniae to epithelial cells transiently expressing RSV G glycoprotein was 2- and 2.2-fold higher, respectively, than adhesion to cells transfected with the vector alone $(P<0.01)$. Furthermore, 4.6- and 6.2-fold larger numbers of NTHi and pneumococci bound to cells expressing a membrane-bound full-length RSV G protein than to cells expressing a truncated non-membrane-bound protein $(P \leqslant 0.005)$. Pre-incubating cells expressing membrane-bound $\mathrm{G}$ protein with blocking anti-RSV $\mathrm{G}$ antibodies reduced bacterial adherence by $78-84 \%(P \leqslant 0.005)$. These studies demonstrate that RSV G protein is a receptor for both NTHi and S. pneumoniae. Strategies to prevent this interaction may reduce the incidence of secondary bacterial complications of RSV infection.
\end{abstract}

\section{INTRODUCTION}

Respiratory syncytial virus (RSV) is an important cause of upper and lower respiratory tract infections, particularly of young children and older adults. These infections are often associated with secondary bacterial infections, including otitis media, pneumonia and acute exacerbations of chronic obstructive pulmonary disease (Andrade et al., 1998; Smith et al., 1976). The mechanisms by which RSV predisposes individuals to secondary bacterial infections are not completely understood. RSV infection increases the numbers of nontypeable Haemophilus influenzae (NTHi) and Streptococcus pneumoniae adhering to human respiratory epithelial cells in vitro and NTHi and S. pneumoniae have been isolated more commonly and in larger numbers from patients with antecedent RSV infections than from patients without RSV infection, suggesting that viral infection increases bacterial colonization (Avadhanula et al., 2006a; Rohde et al., 2003; Smith et al., 1976). Certain eukaryotic cell surface receptors for respiratory

Abbreviations: $\mathrm{CHO}$, Chinese hamster ovary; GFP, green fluorescent protein; NTHi, nontypeable Haemophilus influenzae. bacteria such as intercellular adhesion molecule-1 (ICAM1), carcinoembryonic adhesion molecule 1 (CEACAM1) and platelet activating factor receptor (PAFr) are upregulated by RSV infection (Avadhanula et al., 2006a, b). This, however, does not entirely account for increased bacterial colonization, suggesting that additional bacterial ligands are likely to exist. RSV glycoprotein $(G)$ is a type II transmembrane protein that is expressed on the eukaryotic cell surface following RSV infection and that mediates virus attachment to host cells (Levine et al., 1987). These characteristics suggested that RSV G protein might directly or indirectly promote bacterial adhesion.

\section{METHODS}

Bacteria and viruses. RSV (A2) Long strain, NTHi strain 778, a human respiratory tract isolate, and $S$. pneumoniae strain 357, an encapsulated serotype 19 nasopharyngeal isolate, were propagated as described previously (Avadhanula et al., 2006a).

RSV binding to respiratory bacteria. An adsorption assay was used to determine whether free RSV virions bound directly to bacteria. RSV virions (A2 Long strain, $10^{5.66}$ p.f.u.) were incubated with serial 


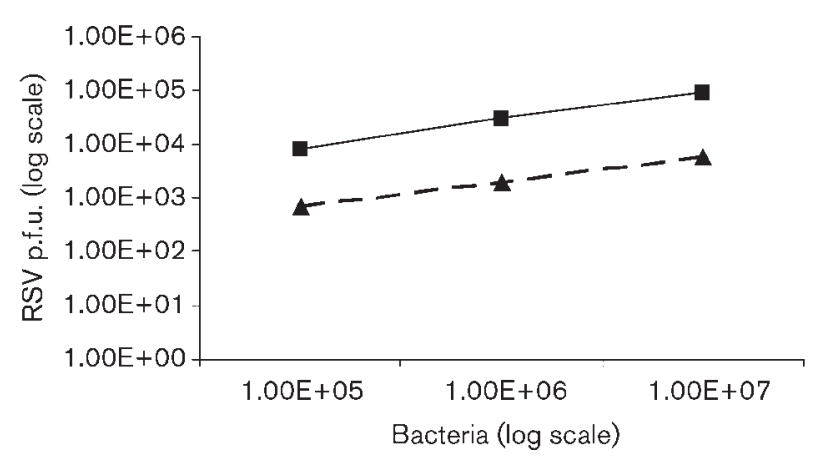

Fig. 1. Adhesion of RSV virions to NTHi and pneumococci. RSV virions were incubated with serial 10-fold dilutions of NTHi (solid line) or S. pneumoniae (dotted line) for $1 \mathrm{~h}$ at $37^{\circ} \mathrm{C}$. Loosely adherent virus was removed by washing and virions associated with bacteria were quantified by plaque assay. Numbers of adherent virions increased with increasing numbers of bacteria.

10-fold dilutions of NTHi or S. pneumoniae for $1 \mathrm{~h}$ at $37^{\circ} \mathrm{C}$. Loosely adherent virus was removed by washing three times with PBS at $3000 \mathrm{~g}$ for $5 \mathrm{~min}$. Virus associated with bacteria was quantified by plaque assay (Kisch \& Johnson, 1963).

Bacterial adhesion to epithelial cells expressing G protein. RSV $\mathrm{G}$ glycoprotein is expressed as a cell membrane-bound type II transmembrane protein and a soluble form lacking the membraneanchor region that originates from an alternative translation initiation codon (Roberts et al., 1994). The coding regions of the full-length (891 bp) and truncated (at M48, $759 \mathrm{bp}$ ) protein were cloned into pCAGGS (Takimoto et al., 2004) or pEGFP-N1 (Clontech) expression vectors. Lipofectamine 2000 (Invitrogen) was used to transfect A549 human respiratory or Chinese hamster ovary $(\mathrm{CHO})$ epithelial cells (American Tissue Type Collection, Mannassas, VA, USA) with pCAGGS encoding full-length RSV G protein (pCAGGS/RSVG), pEGFP-N1 encoding full-length $G$ protein co-expressed with green fluorescent protein (GFP) (pEGFP/RSVG), pEGFP-N1 encoding a truncated $\mathrm{G}$ protein co-expressed with GFP (pEGFP/RSVGt), or the parental vectors. Cells do not secrete GFP expressed in the pEGFP system. G protein expression was determined by FACS analysis and immunofluorescence microscopy using FITC-labelled mouse antiRSV glycoprotein $\mathrm{mAb}\left(\mathrm{IgG}_{3} \kappa ;\right.$ Chemicon International) (Avadhanula et al., 2006a).

In order to obtain a population of cells uniformly expressing $G$ glycoprotein for some studies, $\mathrm{CHO}$ cells were transfected with $20 \mu \mathrm{g}$ pEGFP/RSVG, pEGFP/RSVGt or pEGFP and, $48 \mathrm{~h}$ later, $10^{7}$ cells were sorted using a FACSAria flow cytometer (BD Biosciences) and GFPpositive cells were collected.

Bacteria were grown overnight in liquid broth, diluted $1: 10$ in fresh media and grown to exponential phase as described previously (Avadhanula et al., 2006a). Prior to assays, bacteria were washed three times in PBS and diluted in tissue culture media to $1 \times 10^{6}$ c.f.u. $\mathrm{ml}^{-1}$. Bacterial numbers were determined by $\mathrm{OD}_{600}$ and confirmed retrospectively by plating dilutions on agar plates. For bacterial adherence assays, $5 \times 10^{5}$ epithelial cells were incubated with
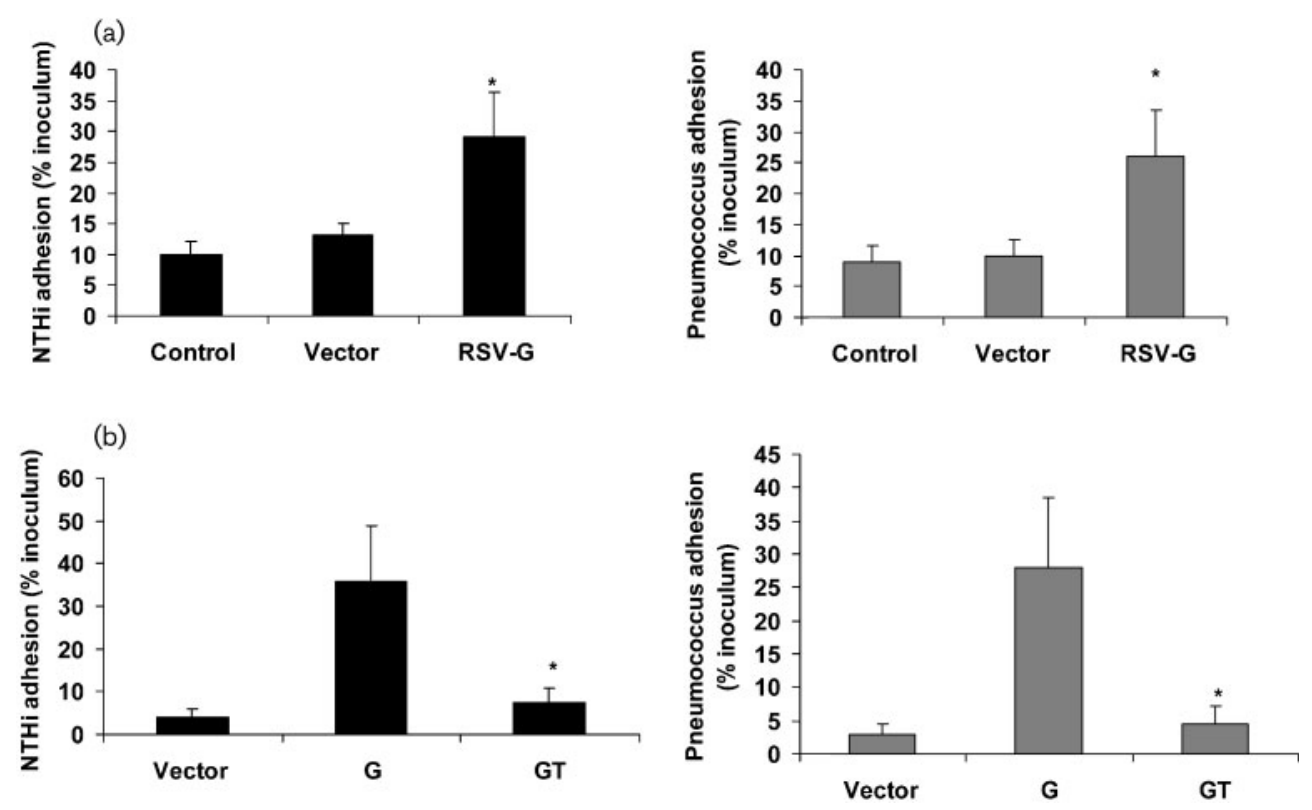

Fig. 2. Adhesion of NTHi and pneumococci to A549 human respiratory epithelial cells and CHO cells transfected with RSV G protein. (a) A549 cells were transfected with pCAGGS/RSVG or pCAGGS vector alone. After $48 \mathrm{~h}$, cells were incubated with NTHi (left, black bars) or pneumococci (right, grey bars) and bacterial adherence was determined. Adherence of NTHi and S. pneumoniae to $A 549$ cells expressing RSV G protein increased significantly compared to cells transfected with vector alone $\left({ }^{*} P\right.$ $<0.05$ ). (b) $\mathrm{CHO}$ cells were transfected with pEGFP/RSVG, pEGFP/RSVGt or the vector alone, incubated with NTHi or pneumococci and bacterial adherence was quantified. Numbers of both NTHi and pneumococci adhering to cells expressing RSV G protein were significantly greater than numbers adhering to cells expressing the truncated Gt protein $\left.{ }^{\star} P<0.005\right)$. Adhesion is expressed as a percentage of the initial inoculum. Data represent the mean $\pm \mathrm{SD}$ of four separate experiments. 
(a)

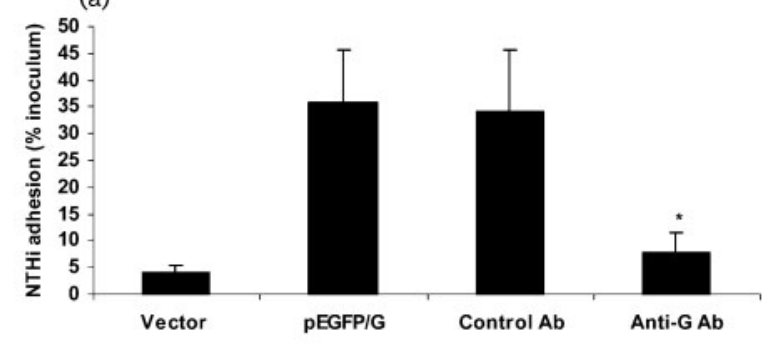

(b)

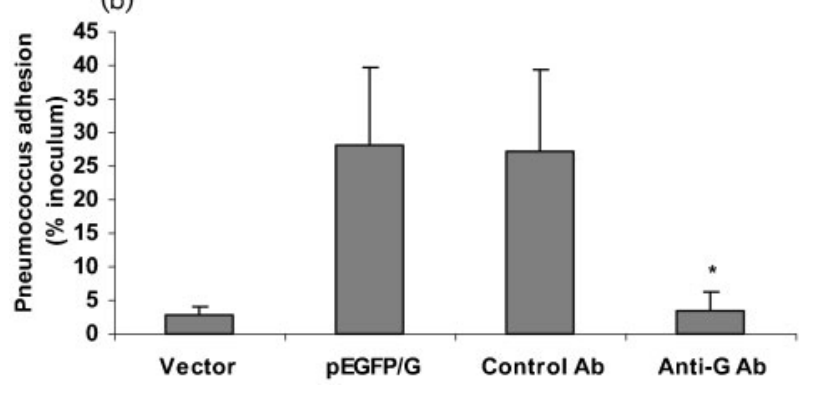

Fig. 3. Blocking eukaryotic cell surface expressed RSV G protein reduces NTHi and pneumococcal adhesion. $\mathrm{CHO}$ cells were transfected with pEGFP/RSV, cell-surface G protein was blocked by incubation with anti-RSV G or control antibody, and numbers of adherent NTHi or $S$. pneumoniae were quantified. Bacterial adhesion to cells expressing full-length $G$ protein significantly decreased following incubation of epithelial cells with anti-G protein, but not with control antibody ( $\left.{ }^{\star} P \leqslant 0.005\right)$. (a) Adhesion of $\mathrm{NTHi}$; (b) adhesion of pneumococci. Data represent the mean \pm SD of three independent experiments.

bacteria (at an m.o.i. of two bacteria per epithelial cell) for $1 \mathrm{~h}$ at $37^{\circ} \mathrm{C}$, washed to remove loosely adherent bacteria, detached by incubation with $1 \times$ trypsin-EDTA (Mediatech) and serial dilutions were plated for quantitative cultures. In each assay, numbers of adherent bacteria were normalized to numbers of epithelial cells (determined in parallel). For assays measuring the inhibition of bacterial adhesion, $\mathrm{CHO}$ cells expressing full-length or truncated $\mathrm{G}$ protein and mock-transfected cells were pre-incubated with $10 \mu \mathrm{g}$ purified mouse anti-RSV $\mathrm{G}$ monoclonal antibody $\mathrm{ml}^{-1}$ (MAB8593; Chemicon International) or an isotype control antibody [J606; anti- $\beta(2 \rightarrow 1)$ fructosan; BD-Biosciences] for $1 \mathrm{~h}$ at $37^{\circ} \mathrm{C}$ prior to addition of bacteria.

Confocal immunofluorescence microscopy was used to co-localize adherent bacteria and cell surface-expressed G protein. NTHi was incubated with FITC $\left(1 \mathrm{mg} \mathrm{ml}^{-1}\right)$ in carbonate buffer containing $0.1 \mathrm{M} \mathrm{NaCl}, 0.09 \mathrm{M} \mathrm{Na}_{2} \mathrm{Co}_{3}$ and $0.015 \mathrm{M} \mathrm{NaHCO}_{3}$ (pH 9.2) for $30 \mathrm{~min}$, washed twice with PBS and resuspended in cell culture media. $\mathrm{CHO}$ cells were propagated on LAB-TEK chamber glass slides, transfected with pCAGGS or pCAGGS/RSVG (Nalge Nunc International) and, after $48 \mathrm{~h}$, inoculated with FITC-labelled NTHi at an m.o.i. of 100 (a higher inoculum than in previous studies was used to better demonstrate bacterial adhesion). Cells were then washed, fixed with $4 \%$ paraformaldehyde (Sigma) for $10 \mathrm{~min}$ at room temperature, and rinsed. After blocking with $4 \%$ normal goat serum (Sigma), $0.5 \%$ BSA and $1 \%$ Triton X-100 in PBS, cells were rinsed with PBS and incubated with a 1/250 dilution of mouse anti-RSV G $\mathrm{mAb}$ (IgG3; Chemicon International) in blocking buffer for $1 \mathrm{~h}$ at $37^{\circ} \mathrm{C}$, then with a $1 / 500$ dilution of Alexa-594-conjugated goat anti-mouse secondary antibody (Molecular Probes) in blocking buffer for $1 \mathrm{~h}$ at $37^{\circ} \mathrm{C}$. After thorough rinsing with PBS, the cells were mounted with TO-PRO-3 nuclear stain (Molecular Probes) and examined with a Leica TCS NT SP confocal laser scanning microscope equipped with argon $(488 \mathrm{~nm})$ and helium-neon $(633 \mathrm{~nm})$ lasers.

Statistical analysis. Data are expressed as the mean \pm standard deviation. Comparison between groups was performed using the Mann-Whitney $U$ test and other data were analysed by Student's $t$ test, with values $P<0.05$ considered significant.

\section{RESULTS AND DISCUSSION}

We hypothesized that NTHi and pneumococci may bind to a viral protein expressed on the eukaryotic cell surface. If this were the case, a linear relationship should exist between the numbers of viruses binding to bacteria following incubation of a fixed number of viruses with increasing numbers of bacteria. In a fluid-phase binding assay, the number of bacterial cell-associated RSV increased directly with increasing numbers of added NTHi or pneumococci (Fig. 1). A total of $10^{3.8}$ p.f.u. bound to $10^{5} \mathrm{NTHi}, 10^{4.3}$ p.f.u. bound to $10^{6}$ bacteria and $10^{4.9}$ p.f.u. bound to $10^{7}$ bacteria. Similarly, a total of $10^{2.7}$ virions bound to $10^{5}$ pneumococci, $10^{3.1}$ virions to $10^{6}$ bacteria and $10^{3.6}$ p.f.u. to $10^{7}$ bacteria, confirming that RSV interacted with a receptor on the bacterial surface. The major RSV surface G glycoprotein, which is expressed on the surface of infected cells and mediates viral attachment to eukaryotic host cells, is a good candidate for such a ligand (Levine et al., 1987).

At $48 \mathrm{~h}$, fewer A549 cells transfected with pCAGGS/RSVG expressed $G$ protein than did cells naturally infected by RSV for $48 \mathrm{~h}(30 \pm 3 \%$ vs $44 \pm 2 \%$, data not shown). Adherence of NTHi to A549 cells transfected with pCAGGS/RSVG, however, was 2.2-fold higher than adherence to cells transfected with the vector only $(P=0.01)$, with $29 \pm 7.3 \%$ of the inoculum ( 0.29 bacteria per cell) adhering to cells expressing $G$ protein, compared to $13 \pm 2.1 \%$ ( 0.13 bacteria per cell) adhering to control cells (Fig. 2). Pneumococcal adherence to A549 cells transfected with pCAGGS/RSVG was similarly increased by 2 -fold $(P=0.001)$. Overall, $20 \pm 7.6 \%$ of the initial inoculum (0.2 bacteria per cell) adhered to pCAGGS/ RSVG-transfected cells compared to $10 \pm 2.6 \%$ of the inoculum ( 0.1 bacteria per cell) adherent to A549 cells transfected with the vector alone.

Previous studies have demonstrated that the larger numbers of NTHi and pneumococci adhering to RSVinfected A549 cells is, in part, attributable to the upregulation of known receptors for NTHi and pneumococci, including ICAM-1, PAFr and CEACAM1 (Avadhanula et al., 2006a). To exclude the possibility that the expression of RSV G protein might augment bacterial adhesion to A549 cells indirectly by upregulating these eukaryotic cell surface receptors, their expression was quantified after transfection of cells with pCAGGS/RSVG or the pCAGGS vector alone. Cells expressing G glycoprotein had similar amounts of surface-expressed ICAM-1, 
CEACAM1 and PAFr as cells transfected with the control vector only at 24,48 or $72 \mathrm{~h}$ after transfection (data not shown), suggesting that $G$ protein directly augments bacteria adhesion. These data are consistent with the observation that blocking bacterial adhesion to RSVinfected epithelial cells with anti-receptor antibodies does not completely reduce excess bacterial adhesion and this implies that additional receptors for NTHi and pneumococci are present on RSV-infected respiratory epithelial cells (Avadhanula et al., 2006a).

The ability of NTHi and pneumococci to bind G protein was confirmed in additional binding and binding inhibition assays. $\mathrm{CHO}$ cells expressing cell surface $\mathrm{G}$ protein or the truncated, non-cell membrane associated $G$ protein and GFP alone were identified and purified by coexpression of GFP to obtain a more homogeneous population of cells expressing a single potential receptor. G protein on $\mathrm{CHO}$ cells transfected with pEGFP/RSVG was observed by immunofluorescence microscopy, but no membrane-bound $\mathrm{G}$ protein was detected on cells transfected with pEGFP/RSVGt, which encodes the secreted form of G only (data not shown). Larger numbers of both NTHi and pneumococci bound to $\mathrm{CHO}$ cells expressing full-length RSV G than to those expressing the secreted $\mathrm{G}$ protein and control cells. For NTHi, $35 \%$ of the starting inoculum ( 0.35 bacteria per cell) bound to $\mathrm{CHO}$ cells expressing RSV G protein compared to only $7.6 \%$ of the inoculum ( 0.076 bacteria per cell) adhering to cells expressing RSVGt, a 4.6-fold smaller number $(P=0.001)$ (Fig. 2). Similarly, $28 \%$ of S. pneumoniae ( 0.28 bacteria per cell) bound to $\mathrm{CHO}$ cells expressing RSV G protein, while only $4.5 \%$ of the inoculum bound to $\mathrm{CHO}$ cells expressing the truncated RSVGt, a 6.2-fold lower number $(P=0.005)$. In the case of both bacteria, adhesion to cells transfected with the non-cell membrane associated truncated G protein did not differ from bacterial adhesion to cells transfected with vectors alone.

In specific blocking assays, adhesion of NTHi was reduced by $78 \%(0.078$ bacteria per cell $)(P=0.005)$ following preincubation of $\mathrm{G}$ protein-expressing $\mathrm{CHO}$ cells with anti-G antibody, and adhesion of pneumococci was reduced by $84 \%$ ( 0.035 bacteria per cell $)(P=0.001)$. Adherence to cells incubated with a control antibody was unchanged (Fig. 3). Adhesion of bacteria to $\mathrm{CHO}$ cells expressing the secreted $G$ protein was low (comparable with adhesion to cells transfected with vector only) and not altered by preincubation with specific or control antibody. Finally, confocal microscopy demonstrated that FITC-labelled NTHi adhered in larger numbers to $\mathrm{CHO}$ cells transiently transfected with RSV G protein than to cells transfected with vector alone and bacteria were predominantly found in association with cells expressing high levels of G (Fig. 4).

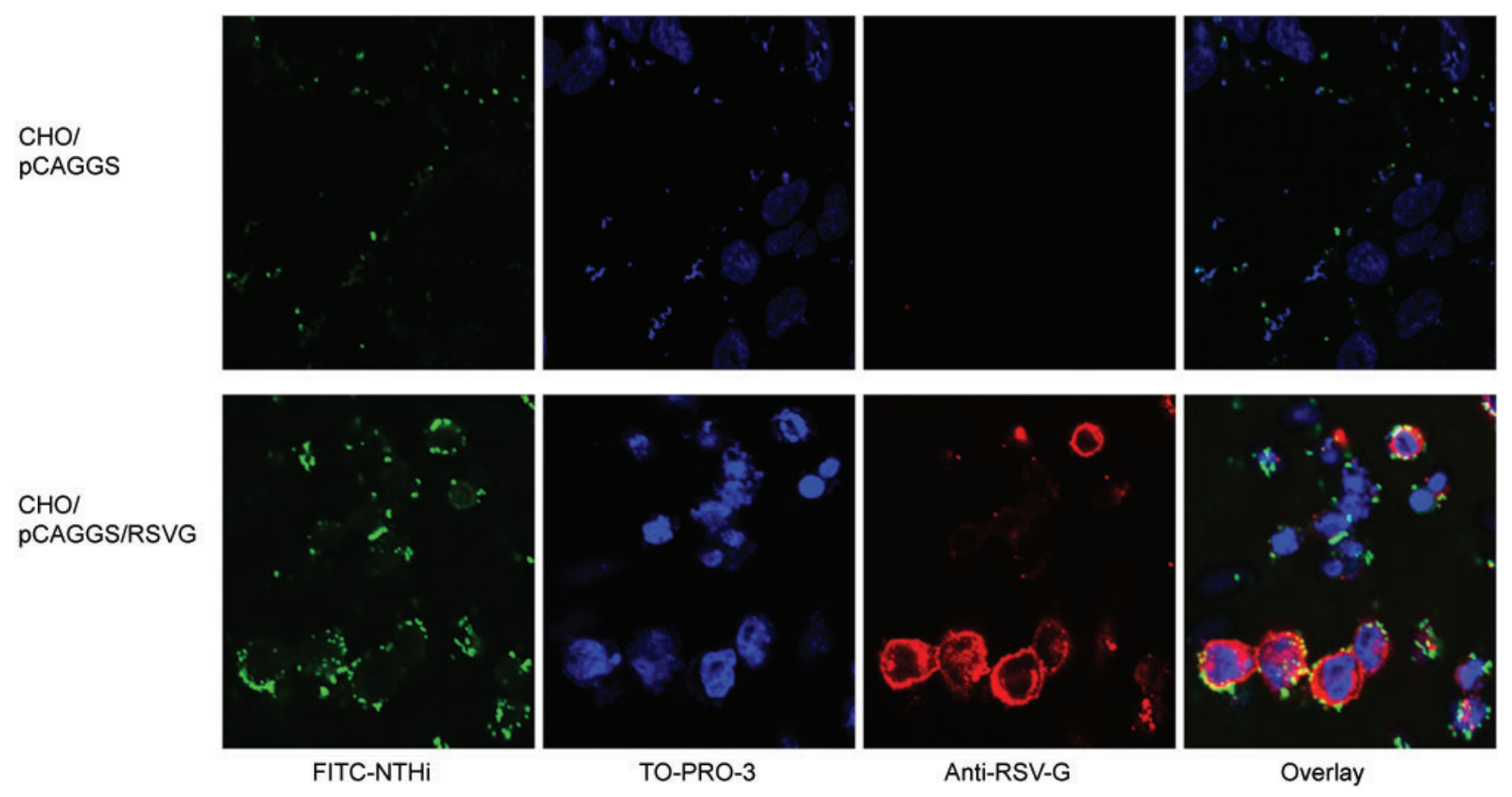

Fig. 4. Interaction of NTHi with cell surface-expressed RSV G protein. CHO cells were transfected with pCAGGS or pCAGGS/RSVG and inoculated with FITC-labelled NTHi (green). Cells were then washed, incubated with mouse anti-RSV G $\mathrm{mAb}$ followed by Alexa-594-conjugated goat anti-mouse secondary antibody, mounted with TO-PRO-3 DNA stain and examined with a confocal laser scanning microscope. TO-PRO-3 stained eukaryotic cell nuclei and NTHi appear blue, and RSV G protein appears red. FITC-labelled NTHi adhered in larger numbers to areas of $\mathrm{CHO}$ cells expressing high levels of $\mathrm{G}$ protein (appearing yellow in overlay) than to vector-transfected cells. 
In summary, NTHi and pneumococci bind to both free RSV virions and epithelial cells transfected with cell membrane-bound $G$ protein, but not to secreted $G$ protein. Bacterial adhesion to $G$ protein-transfected cells is reduced by pre-incubation with specific anti-G antibody, but not an irrelevant control antibody.

Previous studies demonstrated that, at $48 \mathrm{~h}$, approximately sixfold more NTHi and threefold more pneumococci adhered to RSV-infected cells than to uninfected cells (Avadhanula et al., 2006a). RSV infection of respiratory epithelial cells upregulates the expression of several receptors for respiratory pathogens, including ICAM-1, PAFr and CEACAM1, but not respiratory mucin. Inhibiting bacterial interaction with these receptors, however, reduces excess bacterial adhesion to RSV-infected cells by only $70-85 \%$, suggesting that one or more additional receptors for bacteria are expressed de novo or upregulated by RSV infection (Avadhanula et al., 2006a). Our data suggest that RSV G protein, which is expressed on the surface of infected epithelial cells and is required for optimal viral infectivity, is such a receptor.

The G protein ectodomain consists of two mucin-like regions that are structurally highly variable, rich in serine, threonine and proline, and heavily glycosylated with $\mathrm{N}$ and O-linked sugars (Wertz et al., 1985). Adhesion of RSV to eukaryotic cell surface glycosaminoglycans reportedly involves electrostatic interactions between multiple clusters of basic amino acids in the $G$ protein and negatively charged host glycans and proteoglycans (EscribanoRomero et al., 2004). The lipooligosaccharide of NTHi contains $\mathrm{N}$-acetylglucosamine, among other carbohydrates, and the pneumococcal cell wall is composed of repeating units of $\mathrm{N}$-acetylglucosamine and $\mathrm{N}$-acetylmuramic acid (Inzana et al., 1985; Jedrzejas, 2004). It is therefore possible that similar interactions between $G$ protein and bacterial glucosaminoglycans are involved in viral attachment to bacterial cell walls. There is a precedent for the observation that viral envelope proteins expressed on the host cell surface may act as a bacterial receptor. Infection of epithelial cells with influenza virus, for example, results in both the expression of viral haemagglutinin (HA) on the host cell surface and markedly increased numbers of cellassociated Streptococcus pyogenes (Okamoto et al., 1993). Pre-treatment of cells with anti-HA antibody prevented this increase in bacterial adhesion, confirming a specific interaction between bacteria and HA.

Major human respiratory bacterial pathogens use a remarkable diversity and redundancy of strategies to colonize and cause disease. The binding of bacteria to RSV G protein expressed on respiratory epithelial cells is another mechanism used by respiratory bacterial pathogens to adhere to these cells and, potentially, promote local and invasive infection. Further elucidation of the interactions between these important human pathogens may clarify the mechanisms responsible for secondary bacterial infections and lead to strategies to limit these complications.

\section{ACKNOWLEDGEMENTS}

This work was supported by grant CA21765 from the National Institutes of Health and the American Lebanese Syrian Associated Charities (ALSAC).

\section{REFERENCES}

Andrade, M. A., Hoberman, A., Glustein, J., Paradise, J. L. \& Wald, E. R. (1998). Acute otitis media in children with bronchiolitis. Pediatrics 101, 617-619.

Avadhanula, V., Rodriguez, C. A., Devincenzo, J. P., Wang, Y., Webby, R. J., Ulett, G. C. \& Adderson, E. E. (2006a). Respiratory viruses augment the adhesion of bacterial pathogens to respiratory epithelium in a viral species- and cell type-dependent manner. J Virol 80, 1629-1636.

Avadhanula, V., Rodriguez, C. A., Ulett, G. C., Bakaletz, L. O. \& Adderson, E. E. (2006b). Nontypeable Haemophilus influenzae adheres to intercellular adhesion molecule 1 (ICAM-1) on respiratory epithelial cells and upregulates ICAM-1 expression. Infect Immun 74, 830-838.

Escribano-Romero, E., Rawling, J., Garcia-Barreno, B. \& Melero, J. A. (2004). The soluble form of human respiratory syncytial virus attachment protein differs from the membrane-bound form in its oligomeric state but is still capable of binding to cell surface proteoglycans. J Virol 78, 3524-3532.

Inzana, T. J., Seifert, W. E., Jr \& Williams, R. P. (1985). Composition and antigenic activity of the oligosaccharide moiety of Haemophilus influenzae type b lipooligosaccharide. Infect Immun 48, 324-330.

Jedrzejas, M. J. (2004). Extracellular virulence factors of Streptococcus pneumoniae. Front Biosci 9, 891-914.

Kisch, A. L. \& Johnson, K. M. (1963). A plaque assay for respiratory syncytial virus. Proc Soc Exp Biol Med 112, 583-589.

Levine, S., Klaiber-Franco, R. \& Paradiso, P. R. (1987). Demonstration that glycoprotein $G$ is the attachment protein of respiratory syncytial virus. J Gen Virol 68, 2521-2524.

Okamoto, Y., Kudo, K., Ishikawa, K., Ito, E., Togawa, K., Saito, I., Moro, I., Patel, J. A. \& Ogra, P. L. (1993). Presence of respiratory syncytial virus genomic sequences in middle ear fluid and its relationship to expression of cytokines and cell adhesion molecules. J Infect Dis 168, 1277-1281.

Roberts, S. R., Lichtenstein, D., Ball, L. A. \& Wertz, G. W. (1994). The membrane-associated and secreted forms of the respiratory syncytial virus attachment glycoprotein $G$ are synthesized from alternative initiation codons. J Virol 68, 4538-4546.

Rohde, G., Wiethege, A., Borg, I., Kauth, M., Bauer, T. T., Gillissen, A., Bufe, A. \& Schultze-Werninghaus, G. (2003). Respiratory viruses in exacerbations of chronic obstructive pulmonary disease requiring hospitalization: a case-control study. Thorax 58, 37-42.

Smith, C. B., Golden, C., Klauber, M. R., Kanner, R. \& Renzetti, A. (1976). Interactions between viruses and bacteria in patients with chronic bronchitis. J Infect Dis 134, 552-561.

Takimoto, T., Hurwitz, J. L., Coleclough, C., Prouser, C., Krishnamurthy, S., Zhan, X., Boyd, K., Scroggs, R. A., Brown, B. \& other authors (2004). Recombinant Sendai virus expressing the G glycoprotein of respiratory syncytial virus (RSV) elicits immune protection against RSV. J Virol 78, 6043-6047.

Wertz, G. W., Collins, P. L., Huang, Y., Gruber, C., Levine, S. \& Ball, L. A. (1985). Nucleotide sequence of the $G$ protein gene of human respiratory syncytial virus reveals an unusual type of viral membrane protein. Proc Natl Acad Sci U S A 82, 4075-4079. 